\title{
Predictors of serological failure after treatment in HIV-infected patients with early syphilis in the emerging Era of universal antiretroviral therapy use
}

Sadao Jinno ${ }^{1,4^{*}}$, Bryan Anker ${ }^{1}$, Parveen Kaur ${ }^{1}$, Claire C Bristow ${ }^{2}$ and Jeffrey D Klausner ${ }^{3}$

\begin{abstract}
Background: The optimal treatment of early syphilis (primary, secondary and early latent) in HIV-infected patients remains controversial. The Center for Diseases Control STD Treatment Guidelines recommended 1 dose of benzathine penicillin $\mathrm{G}(\mathrm{BPG})$ regardless of HIV infection. However, many providers modify the treatment for early syphilis.

Methods: We performed a retrospective chart review of all cases of early syphilis with positive serologic test results in HIV-infected patients from May 2006 to May 2011 in 2 large, urban HIV clinics. Early syphilis includes primary, secondary, and early latent syphilis. Serological failure was defined as a lack of 4-fold decrease in rapid plasma reagent (RPR) titers 9 to 12 months after syphilis treatment. Patients whose RPR titers decreased after treatment and subsequently increased 4-fold at 9 to 12 months were excluded from the analysis of serological response because of possibility as "reinfection". Baseline characteristics were tested as predictive factors of serological failure using a univariate and multivariate logistic regression model, respectively.
\end{abstract}

Results: Of 560 patients with confirmed cases of early syphilis, 51 (9.0\%) experienced serological failure. Multivariate logistic regression modeling demonstrated that the predictive factors associated with serological failure after early syphilis treatment were baseline RPR titer $\leq 1: 16$ (OR 3.91 [95\% Cl, 2.04-7.47]), a previous history of syphilis (OR 3.12 [95\% Cl, 1.55-6.26]), and a CD4 T-cell count below 350 cells/ml (OR 2.41 [95\% Cl, 1.27-4.56]). Of note, type of syphilis treatment (1 dose versus 3 doses of BPG) did not appear to affect the proportion of serological failure (4\% versus $10 \%, P=0.29$ ), however the power of this study to detect small differences was limited.

Conclusions: HIV-infected patients with baseline RPR titer $\leq 1: 16$, syphilis history, and/or a CD4 T-cell count $<350$ cells/ml should be closely monitored for serologic failure after early syphilis treatment. This study did not detect a substantial difference between treatment with $>1$ dose of BPG and decreased frequency of serological failure, supporting the current recommendation that one dose of BPG is adequate treatment for early syphilis in HIV-infected patients.

Keywords: Syphilis, HIV, Serological failure, Benzathine penicillin

\footnotetext{
* Correspondence: sadaoj@gmail.com

'Department of Medicine, AIDS Healthcare Foundation, Los Angeles, CA, USA

${ }^{4} 1300$ N. Vermont Ave. \#407, Los Angeles, CA 90027, USA

Full list of author information is available at the end of the article
} 


\section{Background}

Despite a significant decrease in syphilis cases in the United States during the 1990s, syphilis incidence has risen dramatically since 2000 [1]. Its prevalence remains especially high in HIV-infected men who have sex with men (MSM). Previous studies demonstrated that HIVinfected patients with syphilis may be more likely to experience serological failure as compared to non-HIVinfected patients $[2,3]$. A recent survey reveals that infectious disease specialists often treat secondary syphilis among HIV-infected patients with 3 doses of benzathine penicillin $\mathrm{G}$ (BPG) rather than 1 dose of BPG (62\% vs $32 \%)$ even though the 2010 Centers for Disease Control and Prevention (CDC) STD Treatment Guidelines recommended treatment for early syphilis remains a single 2.4 million units intramuscular dose of BPG in both HIVinfected and HIV-uninfected patients $[4,5]$. The practice of 3 doses of BPG injection for HIV-infected patients with early syphilis may be motivated by the concern that 1 dose of BPG treatment might result in worse serological and/or clinical complications including central nervous system involvement. However, these approaches have theoretical benefit only, with no evidence of significant clinical benefit vis-à-vis the added cost.

Several studies have demonstrated that certain risk factors are predictive of an increased risk for serological failure [6-8]. For example, Ghanaem et al. found that a CD4 cell count of $<200$ cells/ml and/or the lack of antiretroviral therapy (ART) was associated with an increased risk of serological failure rates among HIV-infected patients with syphilis [6]. Others have demonstrated that baseline rapid plasma reagin (RPR) titers, syphilis stage, age, and number of sex partners may be associated with an increased risk of serological failure in HIVnegative patients with early syphilis $[7,8]$. However, minimal information is available for other risk factors such as treatment regimens, especially a total dose of $\mathrm{BPG}$, or the degree of immnosuppresion in the emerging era of universal ART use. Therefore, we hypothesized that treatment regimens and immunosuppression might contribute to serological response in HIV-infected patients who are on ART. The objective of this study was to assess clinical characteristics and treatment regimens associated with serological response among HIV-infected patients with early (primary, secondary and early latent) syphilis.

\section{Methods}

\section{Setting}

AIDS Healthcare Foundation is one of the largest community-based HIV/AIDS medical providers in the nation, serving over 5000 patients in Los Angeles, California, USA. We performed a retrospective review of medical records to identify all cases of early syphilis with positive serologic test results in HIV-infected patients from
May 2006 to May 2011 in our 2 large urban clinics in Los Angeles. The AIDS Healthcare Foundation human research protection approved the project. During the study period, RPR testing was performed on all patients every 3 months. Inclusion criteria are as follows: (1) serologic follow-up performed 9 to 12 months after syphilis treatment. (2) RPR titers are $\geq 1: 4$ at the time of diagnosis. (3) Clear documentation of the patient's early syphilis stage as primary, secondary or early latent syphilis and type of syphilis therapy received within 2 weeks of diagnosis. (4) In patients with syphilis history, a more than 4 fold increase of RPR titer was required to diagnose a new infection. (5) Positive RPR results were confirmed using fluorescence treponemal antibody absorption test. Patients were excluded from the analysis for the following reasons: no follow-up testing within the period, no documentation of treatment, patients with diagnosis of late latent syphilis or syphilis of unknown duration, cases of primary syphilis with nonreactive serologic characteristics at the time of treatment. For those who satisfied inclusion criteria, medical record review was further performed to obtain information about patient characteristics, medical conditions, age, race, gender, syphilis stage (primary, secondary, early latent), baseline RPR titer, CD4 T-cell count, plasma HIV viral load, ART use, syphilis history, current amphetamine use, syphilis treatment type such as 1 or more doses of BPG, and history of other sexually transmitted diseases. CD4 T-cell count and plasma HIV viral load were analyzed at the time of syphilis diagnosis. CD4 T-cell count, HIV viral load, and age were evaluated as a dichotomous variable (CD4 T-cell count $<350$ cells/ml or $\geq 350$ cells $/ \mathrm{ml}$, detectable HIV viral load as $\geq 400$ copies/ml or undetectable $<400$ copies $/ \mathrm{ml}$, age $\geq 40$ or $<40$ ). ART use was defined as the use of at least 3 antiretroviral drugs, from 2 different classes for a duration of $>6$ months.

Disease stage was classified on the bases of patient history and clinical examination. Patients were considered to have primary syphilis (ulcer at anogenital or oropharyngeal sites and positive serology), secondary syphilis (mucocutaneous and/or skin lesions typical for syphilis and positive serology), or early latent syphilis (no clinical sings of syphilis and newly positive serology within the past year or $\geq 4$-fold increase in RPR titer within the past year).

\section{Study outcomes/definition of serological failure}

The primary outcome was response to syphilis treatment, determined on the basis of change in RPR titers after treatment. Serological failure was defined as a lack of a 4fold decrease in RPR titers at 9 to 12 months after initial treatment or a titer pattern consistent with re-infection. Because we wanted to include only patients likely to have had true treatment failure, patients whose RPR titers decreased after treatment and subsequently increased 4-fold 
at 9 to 12 months were excluded from the analysis of serological response as "reinfection".

\section{Data analysis}

To identify potential predictors of developing serologic failure, we compared demographic and clinical characteristics of patients with serological failure to those with serological cure using univariate logistic regression. Demographic and clinical characteristics for serological failure were selected a priori based on previous literature, clinical plausibility, and ease of clinical collection. Combination of risk factors significant in univariate analyses were assessed and were selected in a stepwise algorithm, with variables entered and excluded for $\mathrm{P}>.025$. Post-hoc power analysis tests were performed, with an alpha of .05, in order to assess the minimum level of effect that would be detected. Data were analyzed using SAS, version 9.3 (SAS Institute).

\section{Results}

During the study period, a total of 777 HIV-infected patients with early syphilis were identified. Among the 777 patients, 217 were excluded due to: lack of data (84 patients), loss of follow-up (48 patients), incomplete chart (37 patients), reinfection (28 patients), or because RPR titer were 1:4 (20 patients). "Reinfection" was defined as a RPR pattern of decreased RPR titers after treatment and subsequently 4 -fold increase between 9 to 12 months.

Table 1 shows the characteristics of the 560 patients included in the analysis. Of 560 persons with confirmed cases of early syphilis, 486 (87\%) were on ART; $96 \%$ of patients were MSM; $51 \%$ had syphilis history; $32 \%$ uses methamphetamine actively; $21 \%$ had hepatitis B or C; $72 \%$ achieved undetectable viral load as defined below 400 copies $/ \mathrm{ml}$. The median CD4 T-cell count was 491. Most (60\%) presented with early latent syphilis. The median RPR titer was 1:64. Majority of patients (78\%) received 3 doses of BPG injection. A total of 51 patients (9.0\%) experienced serological failure.

Table 2 shows the characteristics of the 560 patients included in the univariate analysis. By univariate logistic regression analysis, multiple factors were associated with serological failure, including race (black), syphilis history, RPR titers $\leq 1: 16$, and a CD4 T-cell count $<350$ cells $/ \mathrm{ml}$. There was a risk difference of $-5.6 \%(\mathrm{P}=0.29,95 \% \mathrm{CI}$ : $-12.1,0.8$ ) between the risk of serological failure between the two groups of BPG for syphilis treatment (1 dose versus 3 doses of BPG). However, post hoc power analysis identified based on our observed allocation of BPG treatment (47 patients received 1 dose of BPG; 434 patients received 3 doses of BPG), demonstrated that a statistically significant difference would only be observed with a risk difference of about $13.5 \%$ or greater. Syphilis stage,
Table 1 Clinical characteristics of 560 HIV-infected patients with early syphilis

\begin{tabular}{|c|c|}
\hline Characteristic & No. (\%) \\
\hline Age, median year [IQR] & $40[34,45]$ \\
\hline Male sex & $556(99)$ \\
\hline \multicolumn{2}{|l|}{ Race } \\
\hline Hispanic & $249(44)$ \\
\hline White & $214(38)$ \\
\hline Black & $62(11)$ \\
\hline Other/Unknown & $35(6)$ \\
\hline MSM & $541(96)$ \\
\hline Methamphetamine use & $191(34)$ \\
\hline Hepatitis B or C & $115(21)$ \\
\hline Syphilis history & $287(51)$ \\
\hline Other STD ${ }^{a}$ & $419(75)$ \\
\hline Median RPR titers [IQR] & $1: 64[1: 32,1: 128]$ \\
\hline$<1: 32$ & $134(24)$ \\
\hline$\geq 1: 32$ & $426(76)$ \\
\hline ART & $486(87)$ \\
\hline \multicolumn{2}{|l|}{ CD4 T-cell count } \\
\hline Median CD4 T-cell count, cells/ml [IQR] & $491[345,683]$ \\
\hline$<350$ cells/mL & $144(26)$ \\
\hline$\geq 350$ cells $/ \mathrm{mL}$ & $415(74)$ \\
\hline \multicolumn{2}{|l|}{ HIV RNA } \\
\hline Median HIV RNA level, copies/ml [IQR] & ND $[N D, 631]$ \\
\hline Not detectable ${ }^{b}$ & $400(72)$ \\
\hline Detectable & $160(28)$ \\
\hline \multicolumn{2}{|l|}{ Early syphilis stage } \\
\hline Primary & $77(14)$ \\
\hline Secondary & $146(26)$ \\
\hline Early latent & $337(60)$ \\
\hline \multicolumn{2}{|l|}{ Treatment regimen } \\
\hline 3 doses of benzathine penicillin & $434(78)$ \\
\hline 1 dose of benzathine penicillin & $47(8.4)$ \\
\hline Doxycycline & $78(14)$ \\
\hline Azithromycin & $1(0.2)$ \\
\hline
\end{tabular}

Data are number (\%) of patients, unless otherwise indicated. IQR, interquartile range; MSM, men who have sex with men; RPR, rapid plasma regain test; STD, sexually transmitted disease; HIV, human immunodeficiency virus; DM, diabetes mellitus; ART, combination antiretroviral therapy; ND, not detectable. ${ }^{a}$ Other STD include documented Neisseria gonorrhoeae, chlamydia trachomatis, herpes, and genital warts.

${ }^{\mathrm{b}}$ Defined as $<400$ copies $/ \mathrm{mL}$.

methamphetamine use, age, ART use, and HIV viral load were not significantly associated with serological failure. Neither hepatitis B or C infection was associated with serological failure (odds ratio [OR] 1.07; 95\% confidence interval $[\mathrm{CI}], .53-2.16)$. 
Table 2 Clinical characteristics and predictors of serological failure among 560 HIV-infected patients with early syphilis

\begin{tabular}{|c|c|c|c|}
\hline Characteristic & Patients with serological failure $(n=51)$ & Patients with serological cure $(n=509)$ & Odds ratio $(95 \% \mathrm{Cl})$ \\
\hline \multicolumn{4}{|l|}{ Age, years } \\
\hline$<40$ & $24(8.8)$ & $250(91.2)$ & $0.89(.46-1.79)$ \\
\hline$\geq 40$ & $27(9.4)$ & $259(90.6)$ & 1 \\
\hline \multicolumn{4}{|l|}{ Race } \\
\hline Hispanic & $22(8.8)$ & $227(91.2)$ & 1 \\
\hline White & $17(7.9)$ & $197(92.1)$ & $0.89(.46-1.73)$ \\
\hline Black & $11(17.7)$ & $51(82.3)$ & $2.23(1.02-4.88)$ \\
\hline Other/Unknown & $1(2.9)$ & $34(97.1)$ & $0.30(.04-2.33)$ \\
\hline Methamphetamine use & $19(9.9)$ & $172(90.1)$ & $1.16(.64-2.11)$ \\
\hline Hepatitis B or C & $11(9.6)$ & $104(90.4)$ & $1.07(.53-.16)$ \\
\hline Syphilis history & $40(13.8)$ & $249(86.2)$ & $3.80(1.91-7.56)$ \\
\hline \multicolumn{4}{|l|}{ Other } \\
\hline $\mathrm{STD}^{\mathrm{a}}$ & $39(9.3)$ & $380(90.7)$ & $1.10(.56-2.17)$ \\
\hline \multicolumn{4}{|l|}{ RPR titer } \\
\hline$<1: 32$ & $23(17.0)$ & $112(83.0)$ & $2.91(1.61-5.25)$ \\
\hline$\geq 1: 32$ & $28(6.6)$ & $397(93.4)$ & 1 \\
\hline ART & $44(9.1)$ & $442(90.9)$ & $0.95(.41-2.20)$ \\
\hline \multicolumn{4}{|l|}{ CD4 T-cell count } \\
\hline$<350$ cells/mL & $23(16.0)$ & $121(84.0)$ & $2.63(1.46-4.74)$ \\
\hline$\geq 350$ cells $/ \mathrm{mL}$ & $28(6.7)$ & $388(93.3)$ & 1 \\
\hline \multicolumn{4}{|l|}{ HIV RNA } \\
\hline Undetectable $\mathrm{e}^{\mathrm{b}}$ & $33(8.2)$ & $369(91.8)$ & 1 \\
\hline Detectable & $18(11.4)$ & $140(88.6)$ & $1.44(.78-2.64)$ \\
\hline \multicolumn{4}{|l|}{ Early syphilis stage } \\
\hline Primary & $9(11.7)$ & $68(88.3)$ & 1 \\
\hline Secondary & $10(6.8)$ & $136(93.2)$ & $0.56(.22-1.43)$ \\
\hline Early latent & $32(9.5)$ & $305(90.5)$ & $0.79(.36-1.74)$ \\
\hline \multicolumn{4}{|l|}{ Treatment regimen } \\
\hline 3 doses of benzathine penicillin & $43(9.9)$ & $391(90.1)$ & 1 \\
\hline 1 dose of benzathine penicillin & $2(4.3)$ & $45(95.7)$ & $0.40(.10-1.73)$ \\
\hline Doxycycline & $6(7.7)$ & $72(92.3)$ & $0.76(.31-1.85)$ \\
\hline Azithromycin & $0(0)$ & $1(100)$ & NA \\
\hline
\end{tabular}

Data are no. (\%) patients. OR, Odds Ratio; Cl, confidence interval; RPR, rapid plasma regain test; STD, sexually transmitted disease; HIV, human immunodeficiency virus; DM, diabetes mellitus; ART, antiretroviral therapy.

${ }^{\mathrm{a}}$ Other STD include documented Neisseria gonorrhoeae, Chlamydia trachomatis, herpes, and genital warts.

${ }^{\mathrm{b}}$ Defined as $<400$ copies $/ \mathrm{mL}$.

Using a $P$ value cutoff of .025 , a model identifying serological failure was generated. This multivariate logistic regression modeling demonstrated that the predictive factors associated with serological failure after early syphilis treatment were a CD4 T-cell count below 350 cells/ml (OR 2.41; 95\% [95\% CI, 1.27-4.56]), syphilis history (OR 3.12 [95\% CI, 1.55-6.26]), and baseline RPR titer $\leq 1: 16$ (OR 3.91 [95\% CI, 2.04-7.47]), independent of other characteristics.

\section{Discussion}

Our study was the large systematic evaluation assessing type of syphilis treatment associated with serological response in HIV-infected patients with early syphilis. Our population had a very high risk of past and current syphilis exposure as half had a history of syphilis infection. We found that overall rate of serological failure after therapy was low (9\%) in our HIV-infected patients, and the duration of syphilis treatment ( 1 dose versus 3 doses of 
BPG) did not affect the proportion of serological failure (4\% in a group of 1 dose of BPG versus $10 \%$ in a group of 3 doses of BPG) while our small number of serologic failure limited the power to detect a small difference in serological failure rates between individual who received one dose and three doses of BPG. However, based on our sample size and treatment distribution, we can exclude the difference greater than $13.5 \%$. Our results have important clinical implications therefore it is important to assess whether there is a true lack of effect. HIV-infected patients with early syphilis often receive 3 doses of BPG. However, BPG may have adverse effects including allergic reaction, neurotoxicity, neutropenia, and local pain [9-13]. Therefore, keeping the dose of 2.4 million units of BPG as per the CDC guidelines may be beneficial.

Limited data is available regarding a total dose of BPG for early syphilis in HIV-infected patients, possibly causing substantial variation in the management of early syphilis for HIV-infected patients among clinicians [4]. In preART era, a randomized trial compared 2.4 MU of BPG to enhanced therapy with a 10-day course amoxicillin and probenecid for early syphilis among HIV-infected and non HIV-infected patients [14]. The rates of serological failure did not differ according to treatment group, but it is not entirely clear whether the rate of serological failure differed in HIV-infected patients since most of participants (81\%) were not HIV-infected. More recently, a retrospective study from England, involving $77 \mathrm{HIV}$-infected patients with early syphilis, described that 3 doses of BPG does not significantly alter the serological cure rate as compared to 1 dose. However, the study was underpowered with a result of a wide confidence interval $(78.9 \%$ (95\% CI 68.0 - 89.8) versus $64.1 \%$ (95\% CI 45.0 - 73.2), $P>0.05)$, and did not exclude patients who had been lost to follow-up and/or re-infected. Furthermore, the majority of previous studies suggested that HIV status is not associated with serological response to therapy [3,7,15-19]. For example, a large scale cohort study conducted in Zambia and Rwanda, involving 933 HIVinfected and 388 HIV-uninfected patients, demonstrated that HIV infection did not impact the likelihood of serologic response to therapy in a multivariate analysis (OR, 1.001; $P=$.995) [15]. Their median CD4 T-cell count was 367 cells/ml in Zambia and 446 cells/ml in Rwanda. Although there is no randomized controlled trial comparing therapy of 1 dose with $>1$ doses of BPG in HIV-infected patients with early syphilis, our findings and the review of existing evidence support that one dose of BPG is adequate treatment.

Of interest is our finding that persons with CD4 T-cell count $<350$ cells $/ \mathrm{ml}$ were more likely to develop serological failure, independent of type of treatment (OR 2.41 [95\% CI, 1.27-4.56]). This finding is consistent with previous studies that demonstrated HIV-co-infected patients with low CD4 T-cell count showed significantly slower treatment response [6,7]. We speculate that HIV-infected patients with more immunosuppression may respond to treatment slowly rather than experiencing treatment failure. In fact, among the 23 patients with CD4 T-cell count $<350$ cells/ml who developed serological failure, 20 (87\%) achieved serological cure 12-24 months after treatment, and we did not observe central nervous involvement in 15 out of the 20 patients after evaluation of cerebral fluid examination (5 patients refused cerebrospinal fluid examination).

One of the strongest predictors of developing serological failure in our cohort was RPR titer $\leq 1: 16$ (OR 3.91 [95\% CI, 2.04-7.47]). This finding agrees with the observation that patients with low titers may be at risk of serological failure after treatment as alluded by Sena et al. in HIV negative populations [8]; whereby the authors postulated that high VDRL titers may reflect a more robust immunological response associated with better clearance of the organism. Additionally patients with lower titers may have a longer duration of infection and the response to treatment may be delayed as metabolic activity of syphilis could be lower in later stages of infection [20,21]. Or 4-fold decline in titer may simply be easy to occur in patients with higher titers.

Our study has several limitations. First, a selection bias is possible because $28 \%$ of patients were excluded due to lack of data or loss of follow-up. Secondly, the number of patients treated with one dose of BPG was low $(n=47$, $8.4 \%$ ), and thus a lack of statistical power cannot be excluded as the cause of the observed lack of effect of dosage on serological failure. However, the study did have adequate power to detect the risk difference of $13.5 \%$ or greater. Third, our study was based on medical record review and document may have been incomplete. Fourth, we excluded patients with primary syphilis whose serology was negative as well as those whose titer were $<1: 4$. Therefore, our results may not be generalizable to those who have primary syphilis with negative serology or whose titers are $<1: 4$. Finally, unmeasured potential confounding variables must be considered as limitations of all retrospective cohort studies such as inadvertent exposure to other efficacious antibiotics against syphilis.

\section{Conclusion}

In conclusion, HIV-infected patients with baseline RPR titer $\leq 1: 16$, syphilis history, and/or a CD4 T-cell count $<350$ cells/ml should be closely monitored for serological failure after early syphilis treatment. Treatment with $>1$ dose of BPG was not associated with a large difference in frequency of serological failure. Further research must be conducted to evaluate the current recommendation that one dose of BPG is adequate treatment for early syphilis in HIV-infected patients. 


\section{Competing interests}

They authors declare that they have no competing interests.

\section{Authors' contributions}

SJ collected the clinical information of cohort data and participated in the design of the study, performed statistical analysis, and prepared manuscript. BA collected the clinical information of cohort data. PK prepared manuscript. CCB performed statistical analysis and prepared manuscript. JDK conceived of the study, and participated in its design and coordination and helped to draft the manuscript. All authors read and approved the final manuscript.

\section{Acknowledgements}

Our thanks to Jose Morales for his significant contributions.

\section{Author details}

${ }^{1}$ Department of Medicine, AIDS Healthcare Foundation, Los Angeles, CA, USA. ${ }^{2}$ Fielding School of Public Health, University of California, Los Angeles, CA, USA. ${ }^{3}$ Division of Infectious Diseases, Program in Global Health, University of California, Los Angeles, CA, USA. ${ }^{4} 1300$ N. Vermont Ave. \#407, Los Angeles, CA 90027, USA.

Received: 26 September 2013 Accepted: 23 December 2013

Published: 26 December 2013

\section{References}

1. 2011 CDC Sexually Transmitted Diseases Surveillance. http://www.cdc.gov/std/ stats11/syphilis.htm.

2. Gonzalez-Lopez JJ, Guerrero ML, Lujan R, Tostado SF, de Gorgolas M, Requena $L$ : Factors determining serologic response to treatment in patients with syphilis. Clin Infect Dis 2009, 49(10):1505-1511.

3. Manavi K, McMillan A: The outcome of treatment of early latent syphilis and syphilis with undetermined duration in HIV-infected and HIV-uninfected patients. Int J STD AIDS 2007, 18(12):814-818.

4. Dowell D, Polgreen PM, Beekmann SE, Workowski KA, Berman SM, Peterman TA: Dilemmas in the management of syphilis: a survey of infectious diseases experts. Clin Infect Dis 2009, 49(10):1526-1529.

5. Workowski KA, Berman S: Sexually transmitted diseases treatment guidelines. MMWR Recomm Rep 2010, 59(RR-12):1-110.

6. Ghanem KG, Moore RD, Rompalo AM, Erbelding EJ, Zenilman JM, Gebo KA: Antiretroviral therapy is associated with reduced serologic failure rates for syphilis among HIV-infected patients. Clin Infect Dis 2008, 47(2):258-265.

7. Knaute DF, Graf N, Lautenschlager S, Weber R, Bosshard PP: Serological response to treatment of syphilis according to disease stage and HIV status. Clin Infect Dis 2012, 55(12):1615-1622.

8. Sena AC, Wolff M, Martin DH, et al: Predictors of serological cure and Serofast State after treatment in HIV-negative persons with early syphilis. Clin Infect Dis 2011, 53(11):1092-1099.

9. Hook EW 3rd, Behets F, Van Damme K, et al: A phase III equivalence trial of azithromycin versus benzathine penicillin for treatment of early syphilis. J Infect Dis 2010, 201(11):1729-1735.

10. Hook EW 3rd, Martin DH, Stephens J, Smith BS, Smith K: A randomized, comparative pilot study of azithromycin versus benzathine penicillin $\mathrm{G}$ for treatment of early syphilis. Sex Transm Dis 2002, 29(8):486-490.

11. Riedner G, Rusizoka M, Todd J, et al: Single-dose azithromycin versus penicillin $\mathrm{G}$ benzathine for the treatment of early syphilis. N Engl J Med 2005, 353(12):1236-1244

12. Raichle ME, Kutt H, Louis S, McDowell F: Neurotoxicity of intravenously administered penicillin G. Arch Neurol 1971, 25(3):232-239.

13. Neftel KA, Walti M, Spengler $H$, et al: Neutropenia after penicillins: toxic or immune-mediated? Klin Wochenschr 1981, 59(16):877-888.

14. Rolfs RT, Joesoef MR, Hendershot EF, et al: A randomized trial of enhanced therapy for early syphilis in patients with and without human immunodeficiency virus infection. The Syphilis and HIV Study Group. N Engl J Med 1997, 337(5):307-314.

15. Dionne-Odom J, Karita E, Kilembe W, et al: Syphilis treatment response among HIV-discordant couples in Zambia and Rwanda. Clin Infect Dis 2013, 56(12):1829-1837.

16. Farhi D, Benhaddou N, Grange P, et al: Clinical and serologic baseline and follow-up features of syphilis according to HIV status in the post-HAART era. Medicine (Baltimore) 2009, 88(6):331-340.
17. Gourevitch MN, Selwyn PA, Davenny K, et al: Effects of HIV infection on the serologic manifestations and response to treatment of syphilis in intravenous drug users. Ann Intern Med 1993, 118(5):350-355.

18. Hutchinson CM, Hook EW 3rd, Shepherd M, Verley J, Rompalo AM: Altered clinical presentation of early syphilis in patients with human immunodeficiency virus infection. Ann Intern Med 1994, 121(2):94-100.

19. Janier M, Chastang C, Spindler E, et al: A prospective study of the influence of HIV status on the seroreversion of serological tests for syphilis. Dermatology 1999, 198(4):362-369.

20. Cox DL, Akins DR, Porcella SF, Norgard MV, Radolf JD: Treponema pallidum in gel microdroplets: a novel strategy for investigation of treponemal molecular architecture. Mol Microbiol 1995, 15(6):1151-1164.

21. Magnuson HJ, Eagle $H$, Fleischman R: The minimal infectious inoculum of Spirochaeta pallida (Nichols strain) and a consideration of its rate of multiplication in vivo. Am J Syph Gonorrhea Vener Dis 1948, 32(1):1-18.

doi:10.1186/1471-2334-13-605

Cite this article as: Jinno et al:: Predictors of serological failure after treatment in HIV-infected patients with early syphilis in the emerging Era of universal antiretroviral therapy use. BMC Infectious Diseases 2013 13:605.

\section{Submit your next manuscript to BioMed Central and take full advantage of:}

- Convenient online submission

- Thorough peer review

- No space constraints or color figure charges

- Immediate publication on acceptance

- Inclusion in PubMed, CAS, Scopus and Google Scholar

- Research which is freely available for redistribution

Submit your manuscript at www.biomedcentral.com/submit 